\title{
RESEARCH HIGHLIGHT OPEN DHODH tangoing with GPX4 on the ferroptotic stage
}

\author{
Fudi Wang $\left(D^{1,2}\right.$ and Junxia Min (D) ${ }^{2}$ \\ Signal Transduction and Targeted Therapy (2021)6:244
}

Ferroptosis, an iron-dependent form of regulated cell death, is prevented by activity of the glutathione-dependent phospholipid hydroperoxidase GPX4 (Glutathione peroxidase 4) in the cytosol and mitochondria, and by the glutathione-independent CoQ10 oxidoreductase FSP1 at the plasma membrane. In their recent paper published in Nature, Mao et al. report that $\mathrm{DHODH}$ (Dihydroorotate dehydrogenase) coordinates with GPX4 to block ferroptosis in the mitochondrial inner membrane by reducing ubiquinone to form ubiquinol in cancer cells, thus providing a novel targeted strategy for treating cancer. ${ }^{1}$

Ferroptosis is an iron-dependent, non-apoptotic form of regulated cell death involving lipid peroxidation. ${ }^{2}$ Although the physiological role of ferroptosis has yet to be identified, its pathological functions have been documented in diseases affecting a variety of organs, including the heart, liver, kidney, brain, etc. ${ }^{2,3}$ Notably, compelling evidence supports the notion that targeting genes and pathways that inhibit ferroptosis may provide new opportunities for treating a variety of cancers. ${ }^{2}$ In the past decade, two ferroptosis-inhibiting mechanisms have been identified, involving a wide range of genes and signaling pathways, including the GPX4-driven glutathione-dependent and FSP1-driven CoQ10-dependent metabolic pathways. Importantly, Mao et al. have now linked the mitochondrial enzyme DHODH (dihydroorotate dehydrogenase) to ferroptosis by performing metabolomics analyses in cancer cells treated with the GPX4 inhibitors RSL3 and ML162 to induce ferroptosis (see Fig. 1). ${ }^{1}$

As an iron-containing flavin-dependent enzyme, DHODH plays an essential role in the de novo synthesis of pyrimidines. DHODH catalyzes the conversion of dihydroorotate to orotate via a redox reaction; orotate is then converted to uridine monophosphate, the RNA nucleotide involved in ribosome biogenesis. Based on its essential roles in pyrimidine synthesis and mitochondrial function, $\mathrm{DHODH}$ inhibitors were originally used in the treatment of rheumatoid arthritis and multiple sclerosis. Over half a century ago, the expression and enzymatic activity of DHODH were first associated with cancer progression, yet emerged only recently as a potential therapeutic target for cancer. Since then, considerable effort has been invested in developing DHODH inhibitors and determining their efficacy as well as their anti-cancer and anti-viral potential. However, the mechanisms underlying this enzyme's role in various malignancies and other diseases are poorly understood. To this end, Mao et al. show that ferroptosis is the key mechanism by which inhibiting $\mathrm{DHODH}$ exerts its anti-cancer activity. Their findings suggest that DHODH and mitochondrial GPX4 are two key metabolic enzymes that function to detoxify lipid peroxides that accumulate in the

\author{
; https://doi.org/10.1038/s41392-021-00656-7
}

mitochondria. ${ }^{1}$ Thus, inhibiting both DHODH and GPX4 promotes ferroptosis by increasing lipid peroxidation in the mitochondria, providing a promising strategy for targeting mitochondrial DHODH and GPX4 in cancer cells. In addition, Mao et al.'s data provide compelling evidence that $\mathrm{DHODH}$ inhibitors may be used to treat GPX $4^{\text {low }}$ cancers; moreover, $\mathrm{DHODH}$ inhibitors may be combined with sulfasalazine (an inducer of ferroptosis that inhibits the cystine/glutamate antiporter xCT (SLC7A11/SLC3A2 complex), a component of system $x_{c}{ }^{-}$) in order to treat GPX $4^{\text {high }}$ cancers. Thus, the novel findings presented in their study are clinically relevant and have high translational potential.

The mitochondria are major subcellular organelles involved in the production of reactive oxygen species (ROS) and contain a unique system for regulating iron metabolism. Whether mitochondria are involved in ferroptosis has been unclear; however, emerging evidence supports the notion that mitochondrial damage plays an important role in both the initiation and progression of this iron-dependent form of regulated cell death. Previously, we showed that ferroptosis is the major pathogenic mechanism underlying doxorubicin-induced cardiomyopathy in mice by upregulating $\mathrm{HO}$ 1 (Heme oxygenase-1), causing the production of excess free iron and oxidized lipids in the mitochondrial membrane. Moreover, we found that mitochondria-targeted therapy significantly reduces ferroptosis-induced cardiac injury. ${ }^{3}$

In addition, the previous study reported that the canonical metabolic activity of mitochondria is required for cysteine deprivation-induced ferroptosis. ${ }^{2}$ Thus, the findings are consistent with the notion that mitochondria play an essential role in controlling cell fate by regulating ferroptosis. ${ }^{1}$ Importantly, the role of mitochondria in ferroptosis is tightly regulated by the glutathione-dependent enzyme GPX4 and the glutathioneindependent enzyme DHODH. Because DHODH couples the pyrimidine biosynthesis pathway to the mitochondrial respiratory chain, inhibiting DHODH should affect respiratory chain function. Several important questions remain, however. First, do similar mitochondrial enzymes regulate ferroptosis by generating $\mathrm{CoQH}_{2}$ ? Second, how is mitochondrial iron homeostasis regulated in order to protect against ferroptosis? Thus, further studies are needed to unravel the full picture by which mitochondria contribute to ferroptosis.

In summary, Mao et al.'s study sheds new light on a novel mechanism by which mitochondria prevent ferroptosis, providing important new preclinical evidence suggesting possible strategies for treating GPX $4^{\text {low }}$ cancers by targeting $\mathrm{DHODH}$. Moreover, their study provides a rationale for testing combination therapies in which DHODH inhibitors can be combined with chemotherapy, radiotherapy, and/or immunotherapy,

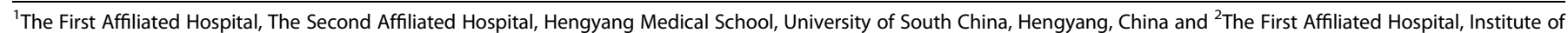
Translational Medicine, Cancer Center, School of Public Health, Zhejiang University School of Medicine, Hangzhou, China

Correspondence: Fudi Wang (fwang@zju.edu.cn) or Junxia Min (Junxiamin@zju.edu.cn)
}

Received: 15 May 2021 Revised: 27 May 2021 Accepted: 27 May 2021

Published online: 18 June 2021 


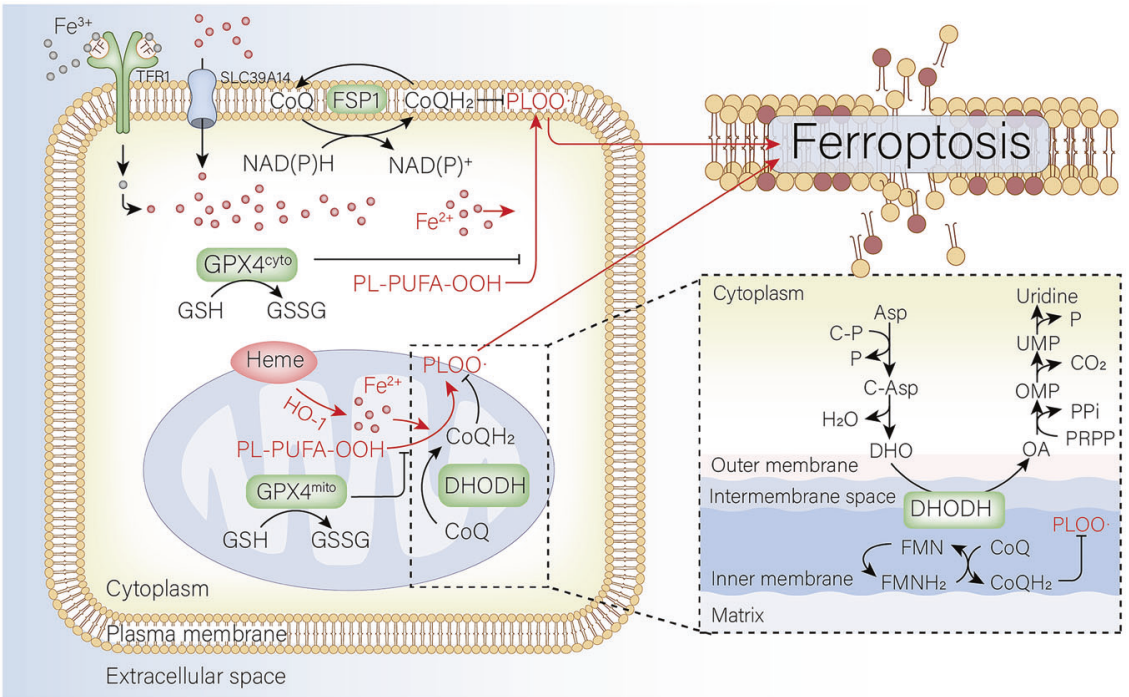

Fig. 1 Schematic model depicting the various pathways in the plasma membrane, cytoplasm, and mitochondria that lead to ferroptosis. Ferroptosis is characterized by iron-dependent cell death with accumulated PLOO. Iron uptake is controlled by TFR1-mediated diferric (Fe3+) transferrin-bound iron and SLC39A14-mediated non-transferrin-bound iron (Fe2+). Whereas PLOO-induced ferroptosis is regulated by either GSH-dependent GPX4 in the cytosol and mitochondria or GSH-independent FSP1 at the plasma membrane. Mao et al. identified DHODH coordinates with GPX4 to inhibit ferroptosis in the mitochondrial inner membrane. PLOO phospholipid hydroperoxyl radical, TFR1 transferrin receptor 1, GSH glutathione, GPX4 glutathione peroxidase 4, FSP1 ferroptosis suppressor protein 1, DHODH dihydroorotate dehydrogenase, CoA coenzyme A, PL-PUFA-OOH phospholipid-polyunsaturated fatty acid-hydroperoxide, GSSG oxidized glutathione, NAD(P)H reduced nicotinamide adenine dinucleotide (phosphate), NAD(P) + oxidized nicotinamide adenine dinucleotide (phosphate)

potentially increasing therapeutic efficacy and improving patient outcome. Despite the promising clinical implications with respect to the use of $\mathrm{DHODH}$ inhibitors, however, ensuring its safety in children is an important concern, given that DHODH deficiency has been linked to Miller syndrome, a rare genetic condition associated with severe micrognathia, cleft lip, eyelid colobomas, supernumerary nipples, and ulnar ray developmental defects. ${ }^{4}$ Another potential caveat is whether the immune system can affect the efficacy of DHODH-based therapies, as DHODH inhibitors were recently tested in a Phase II trial for autoimmune disease and were shown to have antiSARS-CoV-2 and broad-spectrum anti-viral efficacy in vitro. ${ }^{5}$ Nevertheless, future studies are clearly warranted in order to evaluate the feasibility of translating these promising findings into a new strategy for use in the treatment of cancer.

\section{ACKNOWLEDGEMENTS}

We thank Drs. Lianxiang Luo at Guangdong Medical University and Bin Lv at University of South China for helping with drafting the schematic model; and Drs. Xuexian Fang and Jin Li from Wang and Min laboratories for helpful discussion. This work was supported by research grants from the National Natural Science Foundation of China (31930057 to F.W. and 31970689 to J.M.).

\section{ADDITIONAL INFORMATION}

Competing interests: The authors declare no competing interests.

\section{REFERENCES}

1. Mao, C. et al. DHODH-mediated ferroptosis defence is a targetable vulnerability in cancer. Nature 593, 586-590 (2021).

2. Jiang, X., Stockwell, B. R. \& Conrad, M. Ferroptosis: mechanisms, biology and role in disease. Nat. Rev. Mol. Cell Biol. 22, 266-282 (2021).

3. Fang, X. et al. Ferroptosis as a target for protection against cardiomyopathy. Proc. Natl Acad. Sci. USA 116, 2672-2680 (2019).

4. Duley, J. A. et al. Elevated plasma dihydroorotate in Miller syndrome: biochemical, diagnostic and clinical implications, and treatment with uridine. Mol. Genet Metab. 119, 83-90 (2016).

5. Hahn, F. et al. IMU-838, a developmental DHODH inhibitor in phase II for autoimmune disease, shows anti-SARS-CoV-2 and broad-spectrum antiviral efficacy in vitro. Viruses 12, 1394 (2021).

\section{(C)}

Open Access This article is licensed under a Creative Commons Attribution 4.0 International License, which permits use, sharing, adaptation, distribution and reproduction in any medium or format, as long as you give appropriate credit to the original author(s) and the source, provide a link to the Creative Commons license, and indicate if changes were made. The images or other third party material in this article are included in the article's Creative Commons license, unless indicated otherwise in a credit line to the material. If material is not included in the article's Creative Commons license and your intended use is not permitted by statutory regulation or exceeds the permitted use, you will need to obtain permission directly from the copyright holder. To view a copy of this license, visit http://creativecommons. org/licenses/by/4.0/.

(c) The Author(s) 2021 